\title{
Mixture Models: Building a Parameter Space
}

\author{
Vahed Maroufy and Paul Marriott \\ Department of Statistics and Actuarial Science, University of Waterloo
}

\begin{abstract}
Despite the flexibility and popularity of mixture models, their associated parameter spaces are often difficult to represent due to fundamental identification problems. This paper looks at a novel way of representing such a space for general mixtures of exponential families, where the parameters are identifiable, interpretable, and, due to a tractable geometric structure, the space allows fast computational algorithms to be constructed.
\end{abstract}

Keywords: Exponential family, Finite mixture models, Identifiability, Local mixture models, Model selection, Number of components.

\section{Introduction}

Mixtures of exponential family models have found application in almost all areas of statistics, see Lindsay (1995), Everitt (1996), Mclachlan and Peel (2000) and Schlattmann (2009). At their best they can achieve a balance between parsimony, flexibility and interpretability - the ideal of parametric statistical modelling. Despite their ubiquity there are fundamental open problems associated with inference on such models. Since the mixing mechanism is unobserved, a very wide choice of possibilities is always available to the modeller: discrete and finite with known or unknown support, discrete and infinite, continuous, or any plausible combination of these. This gives rise to the first open problem; what is a good way to define a suitable parameter space in this class of models? Other, related, problems include the difficulty of estimating the number of components, possible unboundedness and non-concavity of the log-likelihood function, non-finite Fisher information, and boundary problems giving rise to non-standard analysis. All these issues are described in more detail below. This paper defines a new solution to first of these problems. We show how to construct a parameter space for general mixtures of exponential families, $\int f(x ; \mu) d Q(\mu)$, where the parameters are identifiable, interpretable, and, due to a tractable geometric structure, the space allows fast computational algorithms to be constructed.

\subsection{Background}

Let $f(x ; \mu)$ be a member of the exponential family. It will be convenient, but not essential to any of the results of this paper, to parameterize with the mean parameter $\mu$. We will further assume that the dimension of $\mu$ is small enough to allow underlying Laplace expansions to be reasonable, Shun and McCullagh (1995). A mixture over this family would have the form $\int f(x ; \mu) d Q(\mu)$ where $Q$ is the mixing distribution which, as stated above, can be very general. Since $Q$ may lie in the set of all distributions the 'parameter space' of this set of models is infinite dimensional and very complex. It is tempting to restrict $Q$ to be a finite discrete distribution indeed, as shown by Lindsay (1995), the non-parametric maximum likelihood estimate of $Q$ lies in such a family. Despite this, as the following example clearly shows, this is too rich a class to be identified in a statistically meaningful way.

Example 1 For this example let $f(x ; \mu)=\phi(x ; \mu, 1)$, a normal distribution with unit variance. The QQ plot in Fig. 1 compares two data sets generated from two different finite mixture models with five and ten components respectively. The plot shows that data generated from each can have 
very similar empirical distributions - thus it would be very hard to differentiate between these models and hence estimate the number of components. In this example the components of the mixing distributions have been selected to be close to one another and to have the same lower order moment structure.
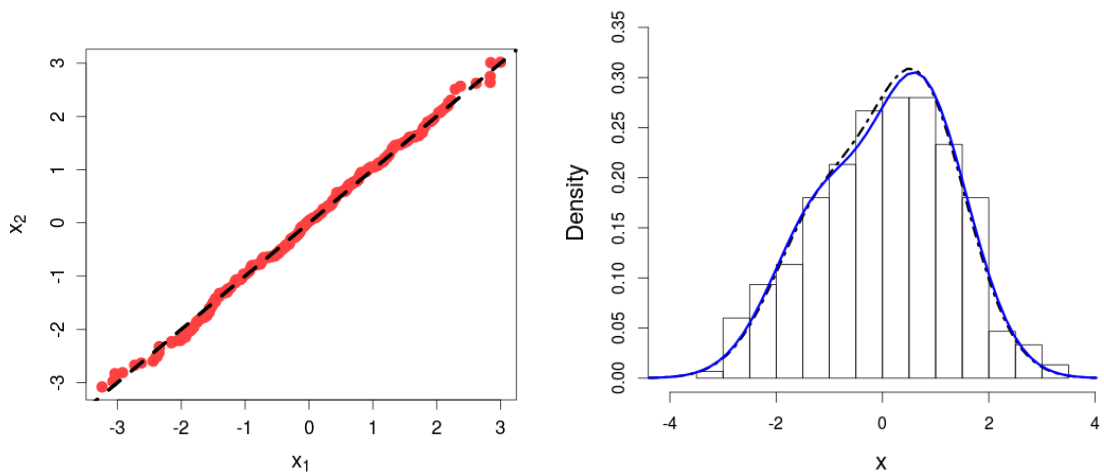

Figure 1: QQ plot, and histogram with fitted density plots

Different methods have been proposed for determining the order of a finite mixture model, including graphical, Bayesian, penalized likelihood maximization, and likelihood ratio hypothesis testing (Mclachlan and Peel, 2000; Hall and Stewart, 2005; Li and Chen, 2010; Maciejowska, 2013). We question though if the order is, fundamentally, an estimable quantity:

(I) first, mixture components may be too close to one another to be resolved with a given set of data, as in Example 1.

(II) Secondly, for any fixed sample size the mixing proportion for some components may be so small that contributions from these components may not be observed.

For instance, Culter and Windham (1994) show, using an extensive simulation, that when the sample size is small or the components are not well separated, likelihood based and penalized likelihood based methods tend to overestimate or underestimate this parameter. Donoho (1988), studies the order as a functional of a mixture density and points out that, "near any distribution of interest, there are empirically indistinguishable distributions (indistinguishable at a given sample size) where the functional takes on arbitrarily large values". He adds, "untestable prior assumptions would be necessary", additional to the empirical data, for placing an upper bound. Celeux (2007) mentions that this problem is weakly identifiable from data as two mixture models with distinct number of components might not be distinguishable.

This fundamental identification issue has immediate consequences when we are trying to define a tractable parameter space. In particular its dimension is problematic: the space will have many dimensional singularities as component points merge or mixing distributions become singular. Identifiability with mixtures has been well studied of course, see Tallis (1969) and Lindsay and Roeder (1993). The boundaries and singularities in the parameter space of a finite mixture have been looked at in Leroux et al. (1992), Chen and Kalbfleisch (1996) and Li et al. (2008) as have the corresponding effects on the shape of the log-likelihood function, for example see Gan and Jiang (1999).

\subsection{The Local Mixture Approach}

Examples where there is a single set of closely grouped components - or the much more general situation where $Q$ is any small-variance distribution - motivated the design of the local mixture model (LMM), Marriott (2002), Anaya-Izquierdo and Marriott (2007). This is constructed around 
parameters about which there is information in the data and can be justified by a Laplace, or Taylor, expansion argument.

Definition 1 For a mean, $\mu$, parametrized density $f(x ; \mu)$ belonging to the regular exponential family, the local mixture of order $k$ is defined as

$$
g_{\mu}(x ; \lambda):=f(x ; \mu)+\sum_{j=1}^{k} \lambda_{j} f^{(j)}(x ; \mu) \quad \lambda \in \Lambda_{\mu} \subset \mathbb{R}^{k}
$$

where $\lambda=\left(\lambda_{1}, \cdots, \lambda_{k}\right)$ and $f^{(j)}(x ; \mu)=\frac{\partial^{j} f}{\partial \mu^{j}}(x ; \mu)$. We denote $q_{j}(x ; \mu):=\frac{f^{(j)}(x ; \mu)}{f(x ; \mu)}$, then for common sample space $S$, and any fixed $\mu$,

$$
\Lambda_{\mu}=\left\{\lambda \mid 1+\sum_{j=1}^{k} \lambda_{j} q_{j}(x ; \mu) \geq 0, \forall x \in S\right\}
$$

is a convex subspace obtained by intersection of half-spaces. The boundary of $\Lambda_{\mu}$ corresponds to a positivity condition on $g_{\mu}(x ; \lambda)$.

Example 2 (1) revisited) The right panel of Fig. 1 shows the LMM fit to the two datasets considered above. We see that the model can successfully capture the shape of the data using only a small number of parameters about which the data is informative.

The local mixture approach is designed, using geometric principles, to generate an excellent inferential frame in the situation which motivated it. The 'cost' associated with these properties is having to work explicitly with boundaries in the inference. We give more details of these properties and the tools associated with working with the boundaries in $\$ 2$ Of course the major weakness of this approach is that it says nothing when the mixing is not 'local'. This paper addresses this issue by looking at finite mixtures of local mixture models. This combines the nice properties of finite mixtures, for example the work of Lindsay (1995), while avoiding the fundamental trap of overidentifying the models as described in $\$ 1.1$. We use this finite mixture of local mixtures to approximate the parameter space of all mixtures. In later sections estimation methods in this very rich model class are discussed, as is the problem of what a particular data set can tell us about the number of components examined in important classes of mixture models.

\section{Local and Global Mixture Models}

Let us consider a general mixture model of the form $\int_{\mu \in M} f(x ; \mu) d Q(\mu)$ where we make the assumption that the support of $Q, M$, is compact. We can therefore partition $M$ as $M=\cup_{i=1}^{L} M_{i}$ where $M_{i} \cap M_{j}=\emptyset$ for $i \neq j$, and each $M_{i}$ is connected. Let us also select a set of 'grid points', $\mu_{i} \in M_{i}$, which will be fixed and known throughout.

The distribution $Q$ can be written as a convex combination of distributions $Q=\sum_{i=1}^{L} \rho_{i} Q_{i}$, where (i) $Q_{i}$ has support $M_{i}$, and (ii) for large enough $L$ each $Q_{i}$ is a localising mixture in the sense required by Anaya-Izquierdo and Marriott (2007), allowing each term $\int_{\mu \in M_{i}} f(x ; \mu) d Q_{i}(\mu)$ to be well approximated by a LMM. In the form given in Definition 1 the mean of the LMM is $\mu+\lambda_{1}$, so there is one degree of ambiguity about the parametrisation $(\mu, \lambda)$. In Anaya-Izquierdo and Marriott (2007) this was resolved by always setting $\lambda_{1}=0$. In Definition 2 the mean ambiguity is resolved by fixing $\mu_{i}$ and having $\lambda_{1}^{i}$ free.

Definition 2 Let $g_{\mu_{l}}\left(x ; \lambda^{l}\right)$ be the LMM from Definition 1 and $\lambda^{l}=\left(\lambda_{1}^{l}, \cdots, \lambda_{k}^{l}\right)$. A discrete mixture of LMMs is defined by

$$
h(x, \mu, \rho, \lambda)=\sum_{l=1}^{L} \rho_{l} g_{\mu_{l}}\left(x ; \lambda^{l}\right)
$$

where $\lambda=\left(\lambda^{1}, \cdots, \lambda^{L}\right), \mu=\left(\mu_{1}, \cdots, \mu_{L}\right)$ is a fixed grid of support points, $\rho=\left(\rho_{1}, \cdots, \rho_{L}\right)$ such that $0 \leq \rho_{l} \leq 1$ and $\sum_{l=1}^{L} \rho_{l}=1$. 
There are some points to consider in this definition. First, the choice of how to select the fixed grid points $\mu_{i}$, in particular how far they should be separated, is clearly critical and discussed in 2.1. Second, throughout this paper we only consider LMMs of order $k=4$. Increasing this degree - while mathematically possible - only adds a small marginal improvement to the local modelling performance, (Marriott. 2006). Third, whenever $f(x ; \mu)$ is a proper exponential family, the terms $q_{j}(x, \mu)$ 's are polynomials of degree $j$, and the interior of the parameter space $\Lambda_{\mu_{0}}$ can be characterized by analysing the roots of a quartic polynomial. Finally, we use throughout two illustrative examples: the normal and binomial.

Example 3 (Normal) For the normal density function $\phi(x ; \mu, 1)$, with fixed variance at $\sigma^{2}=1$, the LMM at $\mu=\mu_{0}$ has the following form,

$$
\begin{aligned}
g_{\mu_{0}}(x ; \lambda)= & \phi\left(x ; \mu_{0}, 1\right)\left\{1+\lambda_{1}\left(x-\mu_{0}\right)+\lambda_{2}\left[\left(x-\mu_{0}\right)^{2}-1\right]+\lambda_{3}\left[\left(x-\mu_{0}\right)^{3}\right.\right. \\
& \left.\left.-3\left(x-\mu_{0}\right)\right]+\lambda_{4}\left[\left(x-\mu_{0}\right)^{4}-6\left(x-\mu_{0}\right)^{2}+3\right]\right\}
\end{aligned}
$$

with,

$$
E(X)=\mu_{0}+\lambda_{1}, \quad \operatorname{Var}_{g}(X)=1+2 \lambda_{2}-\lambda_{1}^{2}, \quad \mu_{g}^{(3)}=6 \lambda_{3}+2 \lambda_{1}^{3}-6 \lambda_{1} \lambda_{2}
$$

in which $\mu_{g}^{(3)}$ is the third central moment. The expression for the first moment and an argument based on Fisher orthogonality of density derivatives (Morris 1982) illustrate how identifiability is attained either by fixing $\mu=\mu_{0}$ or $\lambda_{1}=0$.

Example 4 (Binomial) The LMM for a binomial distribution, with mean $\mu=\mu_{0}$ and number of trials $n$, has a probability mass function of the form

$$
\begin{aligned}
g_{\mu_{0}}(x ; n, \lambda)= & \frac{n ! \mu_{0}^{x}\left(n-\mu_{0}\right)^{n-x}}{x !(n-x) ! n^{n}}\left\{1+\lambda_{1} p_{1}\left(x, \mu_{0}\right)+\lambda_{2} p_{2}\left(x, \mu_{0}\right)+\lambda_{3} p_{3}\left(x, \mu_{0}\right)\right. \\
& \left.+\lambda_{4} p_{4}\left(x, \mu_{0}\right)\right\}
\end{aligned}
$$

where $p_{j}\left(x, \mu_{0}\right)$ is a polynomial with degree $j$. In this example there is extra boundary structure as $\mu$ is limited to the compact set $[0, n]$.

Definition 3 For fixed $\mu_{0}$ the parameter space $\Lambda_{\mu_{0}}$ is a convex subset of $\mathbb{R}^{4}$ and its boundary, $\partial \Lambda_{\mu_{0}}$ is defined by the envelope of hyperplanes

$$
\Pi_{x}:=\left\{\lambda \mid 1+\sum_{j=1}^{4} \lambda_{j} q_{j}(x ; \mu)=0\right\},
$$

parametrized by $x \in S$, Struik (1988).

\subsection{Choosing the Support Points}

In Definition 2 the set of support points, $\left\{\mu_{1}, \cdots, \mu_{L}\right\}$, is assumed fixed and the question remains: how to select it? Recall that the LMM gives a good approximation when the variance of the mixing distribution is small. This would imply that we want neighbouring support points to be close, on the other hand the more support points the larger the value of $L$ and hence the larger the dimension of the parameter space in Definition 2.

The following result follows from standard Taylor remainder results and formalizes the above discussion.

Lemma 1 Suppose $g_{\mu_{0}}(x ; \lambda)$ is the local mixture of the family of densities $f(x ; \mu)$ and $Q$ is a distribution. For any $\delta>0$ there exist an interval $I=\left[\mu_{0}-\epsilon_{1}(\delta), \mu_{0}+\epsilon_{2}(\delta)\right]$ such that

$$
\left|\int_{I} f(x ; \mu) d Q-g_{\mu_{0}}(x ; \lambda)\right|<\delta,
$$

for all $x$. 
Example $5\left(3\right.$ revisited) By Taylor's theorem we have $f(x ; \mu)-g_{\mu_{0}}(x ; \lambda(\mu))=\frac{\left(\mu-\mu_{0}\right)^{5}}{5 !} f^{(5)}(x ; m)$ where $m$ is a value between $\mu$ and $\mu_{0}$. For the normal family with standard deviation $\sigma$ we have

$$
f^{(5)}(y, m)=\left(y^{5}-10 \frac{y^{3}}{\sigma^{2}}+15 \frac{y}{\sigma^{4}}\right) \frac{e^{-\frac{y^{2}}{2 \sigma^{2}}}}{\sqrt{2 \pi} \sigma},
$$

where $y=\frac{(x-m)}{\sigma^{2}}$. This function is obviously bounded, by $M$ say, for all $y \in \mathbb{R}$, and the bound, which only depends on $\sigma$, can be numerically obtained. Letting $\epsilon=\max \left\{\epsilon_{1}, \epsilon_{2}\right\}$ gives,

$$
\begin{aligned}
\left|\int_{I} f(x ; \mu) d Q-\int_{I} g_{\mu_{0}}(x ; \lambda(\mu)) d Q\right| & \leq \int_{I}\left|f(x ; \mu)-g_{\mu_{0}}(x ; \lambda)\right| d Q \\
& \leq\left(\epsilon_{1}+\epsilon_{2}\right) \frac{\epsilon^{5}}{5 !} M
\end{aligned}
$$

The result follows since we can write $\int_{I} g_{\mu_{0}}(x ; \lambda(\mu)) d Q$ as a LMM with $\lambda_{i}:=\int \lambda_{i}(\mu) d Q(\mu)$.

Example 6 (4) revisited) For the binomial family, with probability function $p(x ; n, \mu)$, again we want to bound the error by $\frac{\left(\mu-\mu_{0}\right)^{5}}{5 !} M$, say. We have

$$
p^{(5)}(x ; n, m)=p(x ; n, m) q_{5}(x ; n, m)
$$

where $q_{5}(x ; n, m)$ is a polynomial of degree 5 of both $x$ and $m$, which can be written as

$$
q_{5}(x ; n, m)=\frac{1}{(n-m)^{5}} \sum_{j=0}^{5} \gamma(j)\left(\begin{array}{l}
5 \\
j
\end{array}\right)\left(\frac{n}{m}\right)^{j}(-1)^{5-j}
$$

with $\gamma(j)=j !(5-j) !\left(\begin{array}{l}x \\ j\end{array}\right)\left(\begin{array}{l}n-j \\ n-5\end{array}\right)$. It can be shown that uniformly for all $x=0,1, \cdots, n$, $p(x ; n, m) \leq p\left(x^{\star} ; n, m\right)$, where $x^{\star}=\left\lfloor\frac{m(n+1)}{n}\right\rfloor$, and

$$
L(n, m)<q_{5}(x ; n, m)<U(n, m)
$$

where, for all $m \in[0, n]$,

$$
\begin{aligned}
L(n, m) & =-\frac{\gamma(0)}{(n-m)^{5} m^{4}}\left(5 n^{4}+10 n^{2} m^{2}+m^{4}\right) \\
U(n, m) & =\frac{\gamma(0)}{(n-m)^{5} m^{5}}\left(n^{5}+10 n^{3} m^{2}+5 n m^{4}-m^{5}\right)
\end{aligned}
$$

Moreover, it can be shown that

$$
\begin{cases}U(n, m)>|L(n, m)| & \text { if } \quad 0 \leq m \leq \frac{n}{2} \\ U(n, m)<|L(n, m)| & \text { if } \quad \frac{n}{2}<m \leq n\end{cases}
$$

Therefore,

$$
M=\max _{m \in I} q_{5}\left(x^{\star} ; n, m\right)|L(n, m)| \quad \text { or } \quad M=\max _{m \in I} q_{5}\left(x^{\star} ; n, m\right) U(n, m)
$$

which depends on $\mu_{0}, \epsilon_{1}$ and $\epsilon_{2}$.

\subsection{Estimation Methods}

Estimation with a LMM is, in general, straightforward. The parameter space has nice convexity properties and the likelihood is log-concave, see Anaya-Izquierdo and Marriott (2007). In Marriott (2002) Markov Chain Monte Carlo (MCMC) methods are used since boundaries in the parameter space can easily be accommodated by a simple rejection step whenever a parameter value is proposed that lies outside the boundary. Alternatively direct log-likelihood maximization can be 
done exploiting the convexity of the parameter space and the concavity of the objective function (Maroufy and Marriott, 2015).

Adopting these ideas to finite mixtures of LMMs, we can also easily use MCMC methods. However, here we define a new form of Expectation-Maximization (EM) algorithm, described in the Appendix, and applied in Example 7. In this example we look at mixtures of normals, $\phi\left(x ; \mu, \sigma_{0}^{2}\right)$, where grid-points for $\mu$ are selected as discussed in $\$ 2.1$. To understand the selection of $\sigma_{0}^{2}$ by the modeller we return to point (II) of Section 1.1. This makes the case that we can only estimate clusters, and indeed features of such clusters, if there is the associated information in the data. One consequence of that is the well-known phenomenon that infinite likelihoods are attainable in the case where only a single observation has been associated with a normal cluster and the estimated variance is zero. In our approach we take issue (II) seriously and only put in a LMM component when there is enough data to support its inference. In particular we note that the variance of such a component is $\sigma_{0}^{2}+2 \lambda_{2}-\lambda_{1}^{2}$, which will be bounded below, and vary from cluster to cluster. Hence the data can estimate the variance of each cluster as long as it is above our, modeller selected, threshold.

Example 7 (Acidity data) The data includes acidity index measured in 155 lakes in north-central Wisconsin which is analyzed in Mclachlan and Peel (2000) and the references therein. Using likelihood ratio hypothesis testing, the bootstrap estimated p-value supports two or three components at the $5 \%$ or $10 \%$ level of significance, respectively. However, based on a Bayesian method all the values between two and six are equally supported, Richardson and Green, 1997

Here we select the grid-points $\mu^{(0)}=(3.6,4.2,4.8,5.4,6,6.6,7)$, set $\sigma_{l}=0.5$ and $\gamma=$ 0.15 , so that at least 20 observation is assigned to each cluster. The algorithm returns a twocomponent discrete mixture of LMMs with $\hat{\rho}=(0.676,0.324), \mu=(4.2,6.6)$, Figure 2 (left panel). The middle panel shows that if we give a set of slightly different initial grid points, $\mu_{6}^{(0)}=$ 6.4 instead of 6.6, the algorithm returns the same order for the mixture, with $m=(4.2,6.4)$ and $\hat{\rho}=(0.651,0.349)$, (middle panel). In addition, if we let $\sigma_{l}$ 's to take different values, $\sigma_{6}=0.6$, we get the same order with a slightly different fit (right panel).
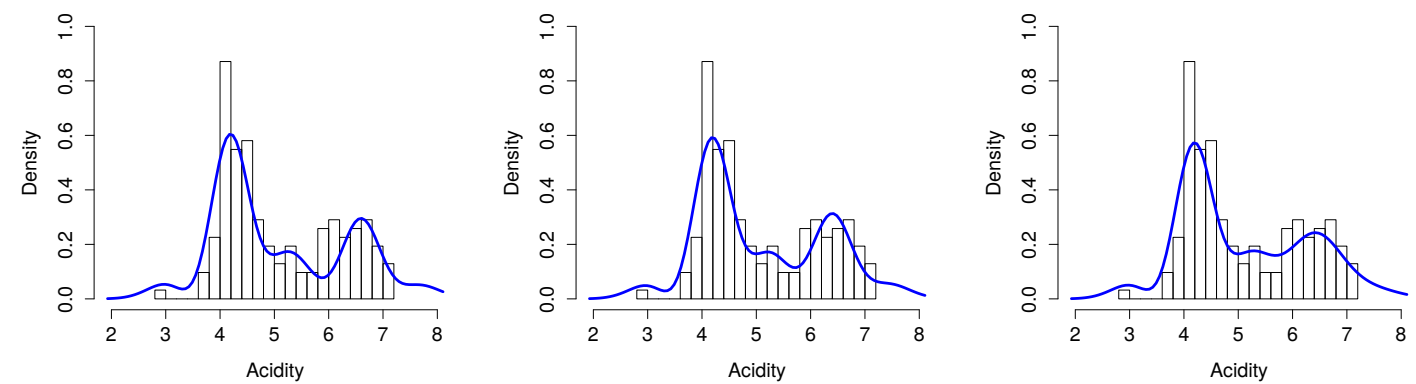

Figure 2: Discrete mixture of LMMs for Acidity data.

Further analysis of the data with different values of $\gamma$, shows how the final results of the algorithm depend on $\gamma$, see Table 1 .

\begin{tabular}{|l|l|l|l|}
\hline$\gamma$ & $\mu$ & $\hat{\rho}$ & Order \\
\hline \hline $0.13,0.14,0.15,0.16,0.17$ & $(4.2,6.6)$ & $(0.67,0.33)$ & 2 \\
\hline $0.1,0.11,0.12$ & $(4.2,4,8,6.6)$ & $(0.57,0.13,0.3)$ & 3 \\
\hline $0.07,0.08,0.09$ & $(4.2,6,6.6)$ & $(0.63,0.18,0.19)$ & 3 \\
\hline 0.06 & $(4.2,4.8,6,6.6)$ & $(0.57,0.08,0.16,0.19)$ & 4 \\
\hline
\end{tabular}

Table 1: Further analysis for different values of $\gamma$ 

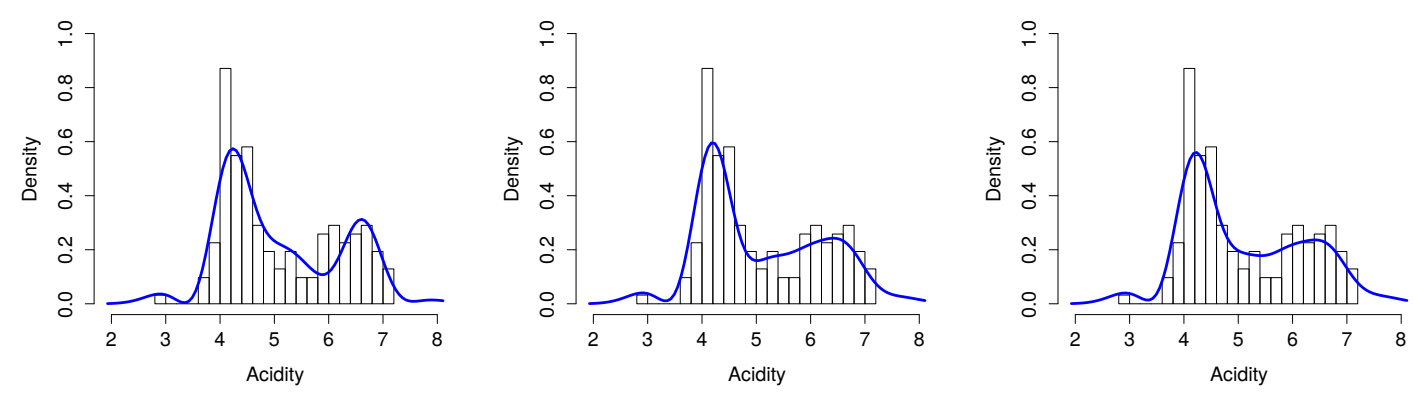

Figure 3: left to right: three and four components fit corresponding to the last three rows in Table 1

\section{Discussion}

While finite mixtures of exponential families are very flexible they suffer from identification problems when support points cluster. This means estimating the order is a very hard problem with a fixed set of data. This paper takes a new approach to this problem. We use a local mixture model to directly model each cluster in a very flexible way. This results in a finite mixture of LMMs. We propose counting these, now well-defined, components as the 'order' - which will now be statistically meaningful. In each of the component LMMs all the parameters are estimable with efficient algorithms where we have applied a principle that we do not considered models which are unestimable from the data at hand.

\section{References}

K. Anaya-Izquierdo and P. Marriott. Local mixture models of exponential families. Bernoulli, 13: 623-640, 2007.

G. Celeux. Mixture models for classification. Advances in Data Analysis, Springer Berlin Heidellberg:3-14, 2007.

J. Chen and J. Kalbfleisch. Penalized minimum-distance estimates in finite mixture models. Canadian Journal of Statistics, 24(2):167-175, 1996.

A. Culter and Windham. Information-based validity functionals for mixture analysis. In Proceedings of the First US/Japan Conference on the frontires of Statistical Modeling in Informational Approach, Amsterdam: Kluwer:149-170, 1994.

D. L. Donoho. One-sided inference about functionals of a density. Annals of statistics, 16:13901420, 1988.

B. S. Everitt. An introduction to finite mixture distributions. Statistical Methods in Medical Research, 5(2):107-127, 1996.

L. Gan and J. Jiang. A test for global maximum. Journal of the American Statistical Association, 94(447):847-854, 1999.

P. Hall and M. Stewart. Theoretical analysis of power in a two-componet normal mixture model. Journal of Statistical Planning and Inference, 134:158-179, 2005.

B. G. Leroux et al. Consistent estimation of a mixing distribution. The Annals of Statistics, 20(3): 1350-1360, 1992.

P. Li and J. Chen. Testing the order of a finite mixture. Journal of the American Statistical Association, 105:491:1084-1092, 2010. 
P. Li, J. Chen, and P. Marriott. Non-finite fisher information and homogeneity: an em approach. Biometrika, pages 1-16, 2008.

B. G. Lindsay. Mixture Models: Theory, Geometry and Applications. Inst of Mathematical Statistics, 1995.

B. G. Lindsay and K. Roeder. Uniqueness of estimation and identifiability in mixture models. Canadian Journal of Statistics, 21(2):139-147, 1993.

K. Maciejowska. Assessing the number of componentsi a normal mixture: an alternative appraoch. University Library of Munich, (No. 50303), 2013.

V. Maroufy and P. Marriott. Generalizing the frailty assumptions in survival analysis. arXiv:1510.02425, 2015.

P. Marriott. On the local geometry of mixture models. Biometrika, 89:77-93, 2002.

P. Marriott. Extending local mixture models. AISM, 59:95-110, 2006.

G. Mclachlan and D. Peel. Finite Mixture Models. John Wiley and sons, 2000.

C. Morris. Natural exponential families with quadratic variance functions. The Annals of Statistics, 10(1):65-80, 1982.

S. Richardson and P. J. Green. On bayesian analysis of mixtures with an unknown number of components (with discuassion). Journal of the Royal Statistical Society B, 59:731-792, 1997.

P. Schlattmann. Medical Applications of Finite Mixture models. Springer, 2009.

Z. Shun and P. McCullagh. Laplace approximation of high dimensional integrals. Journal of the Royal Statistical Society. Series B (Methodological), pages 749-760, 1995.

D. J. Struik. Lectures on Classical Differential Geometry. Dover Publications, 1988.

G. Tallis. The identifiability of mixtures of distributions. Journal of Applied Probability, 6(2): 389-398, 1969.

\section{Appendix}

Starting from initially selected grid points $\mu^{(0)}=\left(\mu_{1}^{(0)}, \cdots, \mu_{L}^{(0)}\right)$, proportions $\rho^{(0)}=\left(\rho_{1}^{(0)}, \cdots, \rho_{L}^{(0)}\right)$ and local mixture parameters $\lambda^{(0)}=\left(\lambda^{1,(0)}, \cdots, \lambda^{L,(0)}\right)$. Suppose we have $\mu^{(r)}$ and $\rho^{(r)}$ and $\lambda^{(r)}$ at step $r$, where $L_{r} \leq L$. For obtaining the estimates at step $r+1$ run the following steps.

1. Calculate $\rho^{(r+1)}=\frac{n_{l}}{n}$, where $n_{l}=\sum_{i=1}^{n} w_{i l}^{(r+1)}$ and

$$
w_{i l}^{(r+1)}=\frac{\rho_{l}^{(r)} g_{\mu_{l}}\left(x_{i}, \lambda^{l,(r)}\right)}{\sum_{l=1}^{L_{r}} \rho_{l}^{(r)} g_{\mu_{l}}\left(x_{i}, \lambda^{l,(r)}\right)}, \quad x=1, \cdots, n ; l=1, \cdots, L_{r}
$$

2. Choose a positive value $0<\gamma<1$, and check if there is any $l$ such that $\rho_{l}^{(r+1)}<\gamma$.

(a) If yes: exclude the components corresponding to $\rho_{l}^{(r+1)}<\gamma$, update $L_{r} \rightarrow L_{r+1}$ and go back to step 1 .

(b) If no: go to step 3 . 
3. Classify the data set into $x^{1}, \cdots x^{L_{r+1}}$ by assigning each $x_{i}$ to only one mixture component. For each $l=1, \cdots, L_{r+1}$, update $\lambda^{l,(r)}$ by

$$
\lambda^{l,(r+1)}=\underset{\lambda \in \Lambda_{\mu_{l}}}{\arg \max } l_{\mu_{l}}\left(x^{l}, \lambda\right),
$$

where $l_{\mu_{l}}\left(x^{l}, \cdot\right)$ is the log-likelihood function for the component $l$.

Remark 1 Step 2 restricts the number of required components for fitting a data set in a way that there is enough information necessary for running inference on each local mixture component. Its value has an influence on the final result of the algorithm in a similar way that an initial value affects the convergence of a general EM algorithm (Table 1). 